Vol. 4 [2021], pp. 109-125 • ISSN: 2659-5311

http://dx.doi.org/10.33776/riesise.v4i1.5164

\title{
LA INNOVACIÓN SOCIAL A TRAVÉS DE LAS REDES DE COLABORACIÓN DE MUJERES PARA EL DESARROLLO SOSTENIBLE: UN ESTUDIO DE LAS REDES LOCALES DE HONDURAS
}

\author{
SOCIAL INNOVATION THOUGHT WOMEN'S \\ COLLABORATION NETWORKS FOR SUSTAINABLE \\ DEVELOPMENT: A STUDY OF LOCAL \\ NETWORK IN HONDURAS
}

María Fernanda Martínez Valladares Universidad Tecnológica Centroamericana. Universidad Anahuac fernanda.martinezva@anahuac.mx

\section{RESUMEN}

El propósito de este artículo es proporcionar un análisis de cómo las redes de colaboración de mujeres en Honduras, dentro y fuera de las empresas, tienen impacto en la innovación social, entendida -de momento- como ideas buenas para el mundo-, el desarrollo sostenible y la solidaridad. Basada en la propia experiencia y estudio de campo de la autora, en este artículo evidencia la maximización del capital social (entendido como incremento del valor) que estas redes generan y su impacto de manera colectiva e individual en las mujeres que las conforman.

\section{PALABRAS CLAVE}

Mujeres; Innovación Social; Solidaridad; Desarrollo; Sostenible; Capital Social; Redes; Círculos Lean In.

\section{Códgos JEL: D63, L39.}

Fecha de recepción: 29/01/2021

\begin{abstract}
The purpose of this article is to provide an analytical approach of how women collaboration networks in Honduras (lean in circle and others), inside and out of the companies, have an impact on social innovation, social development, and solidarity. Base on the experience and field research of the author, this article acknowledges how these networks maximize social capital and has an impact on individual and collective level for the women.
\end{abstract}

KEYWORDS
Women; Innovation; Social; Solidarity; Sustainable Development; Social Capital; Networks; Lean In Circle.

Fecha de aceptación: 22/09/2021 



\section{INTRODUCCIÓN}

Este estudio pretende contribuir a la comprensión de las redes de colaboración de mujeres en Honduras, cómo generan innovación social y cómo contribuyen al cumplimiento de los Objetivos de Desarrollo Sostenible (en adelante ODS) a partir del análisis de 51 entrevistas individuales desarrolladas a mujeres miembros de 3 redes de colaboración hondureñas.

Tomando en cuenta la multiplicidad de debates abiertos sobre género y desarrollo sostenible, este artículo busca responder a la siguiente pregunta: ¿Cómo las redes de colaboración de mujeres contribuyen a los ODS en general y a las mujeres en particular? ¿Podemos afirmar que las organizaciones de mujeres impactan positivamente, aunque sea de una forma simbólica en el desarrollo sostenible?

El propósito es comprender los siguientes aspectos específicos:

a) ¿Cuáles son los asuntos relevantes para las mujeres?

b) ¿Cuáles son los aportes de estas redes para las mujeres como individuos?

c) ¿Cuáles son los aportes de estas redes para la colectividad?

d) ¿Cómo se caracteriza la innovación social promovida por las mjueres en Honduras?

e) ¿Qué impacto tienen las redes colaborativas en la equidad de género?

El artículo se estructura en torno a las siguientes secciones:

En la sección dos se presenta brevemente un Marco teórico, que inicia con la contextualización de la situación de las mujeres en Honduras y las redes de colaboración de mujeres existentes para luego dar paso a los conceptos de: Equidad de género, innovación social, desarrollo sostenible, capital social y redes de colaboración.

En la sección tres se expone brevemente la metodología de la investigación, explicando los criterios de selección de las mujeres y las redes de colaboración y aportando información sobre las entrevistas realizadas.

En la sección cuatro se presentan los resultados de la investigación que responden a las preguntas específicas.

La quinta y última sección aborda las conclusiones y consideraciones finales del trabajo.

Debemos señalar que la investigación y el artículo incluyen el enfoque de género, ya que el contexto actual de la mujer y la equidad de género en 
Honduras son inseparables, considerando que el $51 \%$ de la población que está compuesta por mujeres (INE, 2020) ven afectado su desarrollo humano debido a las diferencias de oportunidades entre hombres y mujeres, mismo que se evidencia en el Indicador sintético de índice de desigualdad de género (PNUD, 2019) que sitúa al país en la posición 116 de 162 países.

\section{MARCO TEÓRICO}

\subsection{CONTEXTO DE LA EQUIDAD DE GÉNERO EN HONDURAS}

\subsubsection{DEFINICIÓN DE EQUIDAD DE GÉNERO}

Pérez y Merino (2009) en su diccionario de definiciones, desarrollam el concepto, vinculando la equidad con la justicia, imparcialidad e igualdad social; Y el género, con la clase que permite agrupar a los seres en características comunes.

Este desarrollo, aporta una visión practica de la equidad de genero como la justicia, imparcialidad e igualdad en temas sociales para un colectivo común.

Según Lampert (2014), para definir la equidad de género tenemos que ir más allá de las diferencias y construir un debate donde se intersecan la diferencia cultural, la problemática de igualdad social, que son parte de los elementos que los Organismos internacionales han utilizado en sus definiciones.

Para la Organización de las Naciones Unidas para la Educación, la Ciencia y la Cultura (UNESCO, 2014), la igualdad de género significa iguales derechos, beneficios, obligaciones, oportunidades, e igual valoración a pesar de diferencias y roles distintos y la equidad implica imparcialidad por lo que es un proceso para lograr la igualdad.

Por su parte, la Comisión Económica para América Latina y El Caribe Equidad de Género (CEPAL, 2014) considera que existe un vínculo indisoluble entre la equidad social y de género con el respeto y ejercicio de los derechos humanos y esto requiere una redistribución de riqueza, el poder y el tiempo

Para la Organización mundial de la salud (OMS, 2002) la lgualdad de Género es la ausencia de discriminación basada en el sexo de la persona en materia de oportunidades, asignación de recursos y beneficios o acceso a los servicios, mientras la Equidad de Género refiere a la imparcialidad y la justicia en la distribución de beneficios y responsabilidades entre hombres mujeres, reconociendo las diferencias en las necesidades y poder con miras a corregir los desequilibrios entre sexos.

Considerando estos conceptos, se puede definir la equidad de género como un enfoque imparcial y justo que busca dotar al hombre y a mujer de las facilidades, derechos y poderes en el plano social, económico y legal, reconociendo las diferencias de roles, necesidades que cada sexo tiene. 


\subsubsection{CONTEXTO HONDURAS}

En Honduras, las mujeres y las niñas representan el $51 \%$ de la población (Instituto Nacional de Estadísticas, 2020). En 1955 las mujeres lograron en su primera conquista política en el país, el voto (PNUD, 2019) y 65 años después según el PNUD (2019) las mujeres son las más afectadas en Honduras ante problemas sociales, económicos, políticos e incluso ambientales.

En su artículo "Sin igualdad de género no se alcanzarán los objetivos de desarrollo sostenible de la agenda 2030" Chaker (2018) pone en manifiesto como no puede un país prosperar y afrontar los obstáculos para el desarrollo sostenible si existe injusticia para más de la mitad de la población del país, en temas de derechos, responsabilidad y oportunidades.

Algunas cifras que demuestran la diferencia de oportunidades en el país son:

Según estudio de la Organización Internacional del Trabajo (OIT, 2020), Honduras es uno de los tres países de América Latina con mayor brecha de género en participación laboral, por cada hombre desocupado, hay dos mujeres desocupadas.

A nivel político, la participación en cargos de elección popular de las mujeres en las últimas elecciones es de $23 \%$ en el Congreso Nacional y solo $8 \%$ son alcaldesas municipales (Tribunal Supremo Electoral, 2017)

En el ámbito empresarial, el Foro Económico Mundial (2020) reconoce que América Latina y el Caribe es una de las regiones peor posicionadas en oportunidad económica femenina, especialmente en niveles de alta dirección. Mismo dato que avala el Consejo Hondureño de Empresa Privada (COHEP, 2019), sólo el $27 \%$ de las mujeres forman parte de las juntas directivas.

Estos y otros indicadores demuestran la necesidad de acciones ante la inequidad de género en el país y en el mundo. En 2015, Naciones Unidas aprobó la agenda 2030, con la cuál la igualdad/ equidad de género tomó un papel más protagónico y se estableció el objetivo 5, lograr la igualdad entre los géneros y empoderar a todas las mujeres y las niñas.

Según el Foro Económico Mundial (2020), existe una brecha de 100 años para alcanzar la equidad de género y por consecuencia para alcanzar el desarrollo sostenible.

\subsection{EQUIDAD DE GÉNERO PARA EL DESARROLLO SOSTENIBLE}

En el informe de Brundtland (ONU, 1987) se define el desarrollo sostenible como "satisfacer nuestras necesidades actuales sin comprometer la capacidad de las generaciones futuras para satisfacer las suyas" lo que implica la protección del planeta, de las personas actuales y futuras de manera equitativa. 
Posterior a esto el concepto siguió nutriéndose en la Cumbre de la Tierra (ONU, 1992), donde referencia la relación entre desarrollo económico, social y ambiental con la reducción de la pobreza y la reducción de desigualdad de niveles de vida.

En 2002 la Comisión Económica para América Latina, reconoce la función que cumple el medio ambiente y los recursos naturales para garantizar el progreso económico.

En su artículo El discurso del desarrollo sostenible, Rodríguez y Govea (2006) capturan los elementos de los distintos organismos y personas que describen el desarrollo sostenible e identifican tres vertientes que aportan un robusto concepto de desarrollo sostenible.

La primera se relaciona con la etimología de la palabra latina Subvenir que significa mantener arriba o apoyar desde abajo, según lo cual una comunidad está apoyada en su historia, sentido de continuidad y arraigo.

La segunda vertiente son los intereses relevantes de las personas en su visión al futuro y el bienestar social, económico y ambiental sumado a los factores políticos para el logro de fines comunes.

La tercera vertiente implica la interrelación persona-sociedad-ambiente, que propone reexaminar el rol de la economía; la concepción sobre la naturaleza; el papel de la ciencia y la tecnología, y los valores sobre los cuales se construye socialmente la conciencia ciudadana y la responsabilidad social.

Considerando las tres vertientes anteriormente mencionadas el concepto de desarrollo sostenible continuó evolucionando, pasando por la Declaración del Milenio del 2000 y para el 25 de septiembre de 2015, Naciones Unidas adopta en conjunto con gobierno, sociedad civil, sector privado una serie de objetivos para erradicar la pobreza, proteger el planeta y asegurar la prosperidad para todos como parte de una nueva agenda común conocidos como Objetivos de Desarrollo Sostenible (Naciones Unidas, 2015).

Los Objetivos de Desarrollo Sostenible no están exceptuados de críticas. Desde la perspectiva jurídica, Cardesa-Salzmann y Pigrau (2017) cuestionan que los ODS no paren aptos para alterar significativamente la fragmentación del ordenamiento Jurídico Internacional Contemporáneo lo que disminuye su valor a nivel de justicia distributiva y genera el riesgo que como sus predecesores los objetivos de desarrollo del milenio, estos no se cumplan.

Por su parte, con una visión más utilitarista, William Easterly (2015), cuestiona el hecho que las iniciativas como los ODS, atienden siempre a la pregunta ¿Qué debemos hacer? Y el por qué las acciones que se recomienda tomar en con los ODS son aquellas que ya se han propuesto en cumbres anteriores y fallado. 
Del lado de los promotores de los ODS, se encuentran líderes del sector privado como Paul Polman, quien en el Foro Económico Mundial en Davos (2016), que su meta ideal era que cada empresa privada del mundo diseñara su plan de negocios y sus objetivos alineados a los ODS. En el mismo año, Christiana Figueres (Naciones Unidas, 2016) menciona en la Conferencia Mundial del Clima, que la adopción de los ODS, son la oportunidad para enfrentar los retos del cambio climático.

Independientemente de si son promotores o detractores de los ODS, es innegable que brindan un marco de referencia para atender las necesidades de desarrollo sostenible entre ellas la equidad de género.

En el apartado 2.1.2 se menciona superficialmente el ODS 5: lograr la igualdad entre los géneros y empoderar a todas las mujeres y las niñas.

Considerando este objetivo ¿Qué relación hay entre el desarrollo sostenible y la equidad de género?

La Agenda 2030 (Naciones Unidas, 2020) entiende la igualdad/ equidad de género no solo como un derecho fundamental, sino que como una necesidad para un mundo pacífico, próspero y sostenible. Reconoce el efecto multiplicador que tienen las mujeres en el desarrollo y como no se alcanzará la sostenibilidad si esta mitad de la población no puede progresar.

Entre los retos que este objetivo plantea están: Acceso a recursos, activos económicos y tierra, acceso a créditos, transferencia de tecnología, financiación relacionada con el clima, mercado laboral, salarios y oportunidades, carga de trabajo no remunerado y necesidades básicas, participación en la toma de decisiones, violencia, repercusiones por el cambio climático, educación.

Para abordar estos retos se necesitan esfuerzos multisectoriales que consideren las experiencias, las necesidades y contribuciones de las mujeres para el desarrollo sostenible, es aquí donde las redes de colaboración y la innovación social sirven de catalizadores para el cambio.

\subsection{REDES DE COLABORACIÓN DE MUJERES:}

INNOVACIÓN QUE GENERA VALOR SOCIAL Y SOLIDARIDAD

\subsubsection{DEFINIENDO LA INNOVACIÓN SOCIAL}

Muñoz y Sánchez (2018) entienden la innovación social como un proceso creativo que surge de las interacciones entre actores organizados, con la finalidad de crear valor público que se traduce en cambio de actitudes, comportamientos, percepciones, rutinas organizacionales y nuevas instituciones que se caracterizan por ser al mismo tiempo financiera y socialmente rentables para el beneficio de la sociedad en general. 
Por su parte Christensen (2006) considera la innovación social como un abordaje de un problema social en una nueva manera, creando soluciones, escalables, sostenibles y que cambien el sistema

Herrero de Egaña (2018) se refiere a la innovación social como formas nuevas o novedosas que tiene la sociedad, de hacer frente a los retos sociales relevantes (RSR), que son más eficaces, eficientes y sostenibles o que generan mayor impacto que las precedentes y que contribuyen a hacerla más fuerte y articulada.

Tomando como referencia la Caracterización de Muñoz y Sánchez (2018) sobre la innovación social, se desarrolla la tabla 1 a manera de resumen conceptual

Tabla 1. Resumen conceptual de la Innovación social

Un abordaje a un problema- Christensen

¿Qué es? Nuevas Formas de hacer las cosas- Herrero de Egaña Un proceso- Muñoz Grande

\begin{tabular}{c|l}
\hline \multirow{2}{*}{$\begin{array}{c}\text { ¿Para qué } \\
\text { surge? }\end{array}$} & $\begin{array}{l}\text { Muñoz Grande } \\
\text { Para hacer frente a los retos sociales relevantes- He- } \\
\text { rrero de Egaña } \\
\text { Para cambiar el sistema- Christensen }\end{array}$ \\
\hline & Ante la necesidad de generar propuestas rentables
\end{tabular}

¿Por qué surge? Grande Por la necesidad de construir una sociedad más fuerte y articulada- Herrero de Egaña

Es rentable financieramente- Muñoz Grande

Es rentable socialmente- Muñoz Grande

Proceso participativo y de trabajo colaborativo- Co-

Características munidad Europea

Escalable- Christensen

Sostenible-Christensen

Eficientes- Herrero de Egaña

Eficaces- Herrero de Egaña

¿Quién la

La sociedad- Herrero de Egaña

instrumenta?

Actores organizados- Muñoz Grande

Fuentes: Elaboración propia 


\subsubsection{REDES COLABORATIVAS, CAPITAL O VALOR SOCIAL Y SOLIDARIDAD}

En el apartado anterior, los tres enfoques presentados de innovación social tienen como factores en común: novedad, solución a problemas sociales, colaboración y sostenibilidad.

Partiendo de las palabras colaboración y solución de problemas, se entenderán las redes de mujeres como: Espacios colaborativos formados por mujeres con intereses en común que se brindan apoyo mutuo para solucionar problemas individuales y colectivos.

Existen muchos tipos de redes colaborativas de mujeres, para efectos de este artículo se consideran las siguientes:

Tabla 2. Tipos de redes de colaboración de mujeres

\begin{tabular}{c|c|c} 
Tipo & ¿Qué son? & Tipos \\
\hline $\begin{array}{c}\text { Círculos Lean } \\
\text { In }\end{array}$ & $\begin{array}{c}\text { Grupos pequeños de mujeres que se } \\
\text { reúnen con frecuencia y se acompa- } \\
\text { ñan para alcanzar sus metas y cons- } \\
\text { truir un mundo mejor (Lean in circle, } \\
\text { 2020) }\end{array}$ & $\begin{array}{c}\text { Corporativos } \\
\text { Sociales }\end{array}$ \\
\hline $\begin{array}{c}\text { Redes de } \\
\text { mentoría }\end{array}$ & $\begin{array}{c}\text { Grupos de mujeres empresarias/ } \\
\text { intercambio de experiencias y cono- } \\
\text { cimientos logran fortalecer sus nego- } \\
\text { cios o ideas. (Voces Vitales, 2020) }\end{array}$ & $\begin{array}{c}\text { Corporativos } \\
\text { Voluntarios }\end{array}$ \\
\hline $\begin{array}{c}\text { Redes aca- } \\
\text { démicas/ } \\
\text { científicas }\end{array}$ & $\begin{array}{c}\text { Foros de mujeres que colaboran para } \\
\text { el desarrollo de sus temas de interés } \\
\text { y desarrollo de habilidades indivi- } \\
\text { duales y colectivas. (OWSD, 2020) }\end{array}$ & Por área \\
\hline Fenerales
\end{tabular}

Fuente: Elaboración propia

Betoni (2011), en su investigación sobre desarrollo, identificó que la capacidad asociativa, cooperación, confianza interpersonal y conciencia cívica que generan las redes de apoyo, facilitan el flujo de información, la coordinación y la toma de decisiones colectivas y que aquellos países que poseen capital social han tenido mejores resultados en termino de desempeño económico y democracia. Las redes de colaboración de mujeres contribuyen desde esta perspectiva al desarrollo sostenible, ya que generan capital social y ¿Qué es el capital social?

Portela y Neira (2003) consideran el capital social o valor social como una acumulación de varios tipos de activos sociales, psicológicos, culturales, 
cognoscitivos, institucionales, etc. que aumentan la probabilidad de un comportamiento cooperativo mutuamente beneficioso para las personas que lo poseen y para la sociedad en general. Este concepto incluye de manera implícita la solidaridad, entendiendo esta como la adhesión a la causa de otros (RAE, 2020).

\section{METODOLOGIA}

Este artículo es resultado de una reflexión individual, de la experiencia de la autora como coordinadora, participante y mentora en redes de mujeres, así como la experiencia en particular de 3 grupos de redes de colaboración de mujeres:

- Capítulo OWSD Honduras

- Círculos lean in en Tegucigalpa

- Mentoras y aprendices de Mujer Acelera a nivel nacional

Se realiza una investigación explicativa, cualitativa, por conveniencia seleccionando miembros los tres grupos antes mencionados.

Se desarrollaron 51 entrevistas en profundidad, de mujeres miembros de alguna de las redes antes mencionadas y se llevaron a cabo procesos de observación e interpretación de las interacciones de las de mujeres.

Durante 2019 y 2020 se observó el comportamiento de los miembros de estas redes y durante 2020 se llevaron a cabo las entrevistas a profundidad.

Las redes fueron seleccionadas ya que son aquellas con la que la autora interactúa y ha observado el comportamiento en años anteriores.

En relación con la selección de las mujeres, se mandó una invitación al $100 \%$ de mujeres activas en estas tres redes que para el momento de la investigación eran: 28 OWSD, 40 mujer acelera y 15 del círculo lean in.

Cabe mencionar que varias de las mujeres pertenecen a uno más de los círculos por lo que el universo de mujeres pertenecientes a las tres comunidades seleccionadas es de 58. Para la fecha en que se realizó la investigación

Se coordinaron entrevistas escritas y por videollamada. Considerando Honduras ya se encontraba en confinamiento pro la pandemia de Covid19, no se realizó ninguna entrevista de forma presencial.

Al lograr 51 entrevistas se tiene un nivel de confianza de 95\% y 5\% de margen de error considerando que la población única de mujeres en estas redes es de 58.

\section{RESULTADOS}

4.1 CARACTERIZACIÓN DE LA INNOVACIÓN SOCIAL VINCULADA A REDES DE MUJERES EN HONDURAS

Utilizando como marco de referencia el sistema de códigos (160 códigos) desarrollado por Blanca Herrero de Egaña para su tesis doctoral "La innovación social en España: ejes vertebradores desde la Teoría Fundamentada" (2018) para caracterizar la innovación social española, 
observamos que la principal característica identificada en la innovadora social hondureña es la sensibilidad social, entendida como la habilidad de un individuo para identificar, percibir y entender las señales y contextos en las interacciones social (Lifeder, 2020), en contraste con esto, se auto percibe como un ser más racional que idealista. Se encuentran similitudes en características como idealista y creativa, en comparación con los resultados obtenidos por Herrero de Egaña (2019) en su investigación sobre la innovación y el emprendimiento sociales de mujeres en España.

Figura 1. Principales características que identifican a las innovadoras sociales

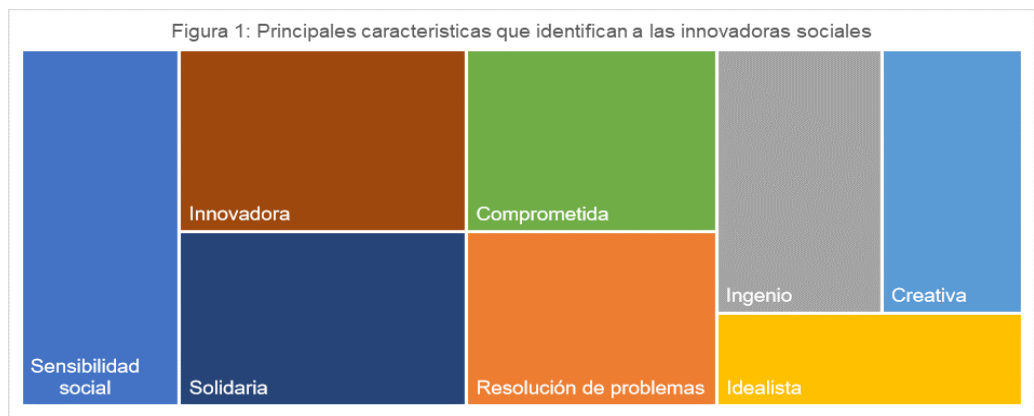

Fuente: Elaboración propia, considerando sistema de códigos de Herrero de Egaña (2018)

Figura 2. Relación laboral por tipo de red

Figura 2: rubro de trabajo por tipo de red a la que pertenece

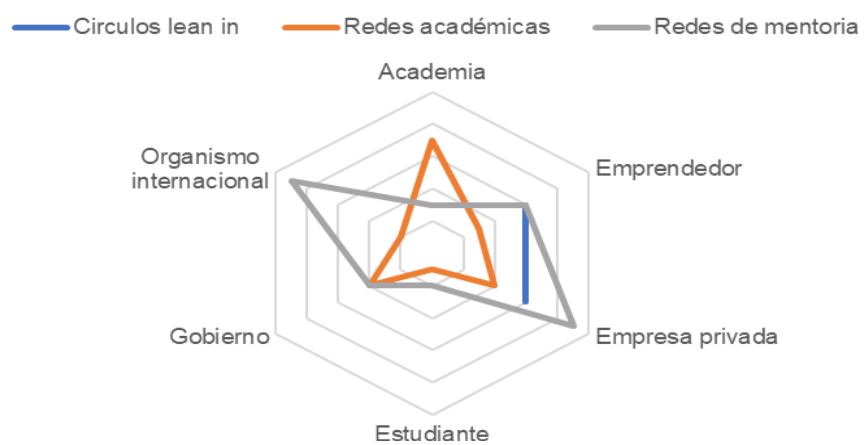

Fuente: Elaboración propia.

La mayoría de mujeres que participaron en la investigación trabajan en el sector privado y participan en redes de mentoría. 
Independientemente el área de trabajo, las redes de mentoría son las de mayor crecimiento, sin embargo, no son las que generan mayor desarrollo.

Al comparar los datos contra la de círculos lean in, se observa que el $100 \%$ de las mujeres miembros de círculos lean in pertenecen al menos a otra red de colaboración de mujeres, así como el $80 \%$ de las miembros de los circulos lean in, afirman haber emprendido con el apoyo de sus compañeras de red, por tanto de los tres tipos identificados de redes de mujeres los círculos lean in parecen que podrían generar un mayor capital social, cumpliendo la promesa de valor de sus fundadoras, quienes aseguran que las mujeres son más capaces de conseguir las metas cuando se trabaja en equipo. Como referencia, existen 35,000 círculos lean in en 160 países. (Lean in circle, 2020). Sin embargo, dado el carácter descriptivo y exploratorio de la presente investigación, estos primeros hallazgos, como hipótesis, deberán ser contrastados en investigación posteriores.

En el caso de las redes académicas, se observa que sólo las estudiantes hoy no están participando en redes académicas, lo que en sí representa una oportunidad por la diversidad de redes que existen dentro de los centros educativos.

Figura 3. Detonantes de su innovación social y participación en redes

¿Cuál es la principal razón por la que te uniste a una red de mujeres?

51 responses
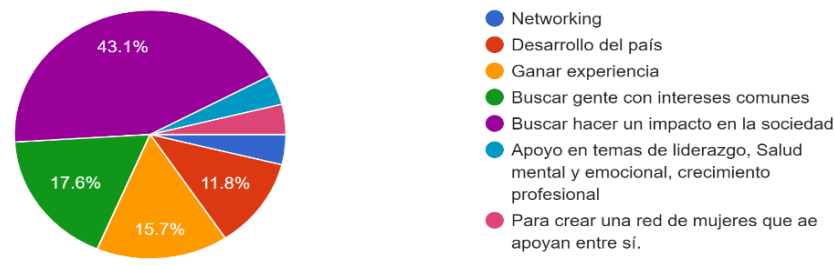

Fuente: Elaboración propia.

Al indagar sobre qué motiva a las mujeres entrevistadas a participar en redes de colaboración de mujeres, el impacto social que su participación en las redes tiene se lleva el primer lugar, seguido de otros motivadores como encontrar personas con intereses en común, ganar experiencia y el desarrollo del país. Esto sin duda refuerza la postura inicial de que las redes de mujeres contribuyen a aportar desarrollo sostenible y pueden ser vistas como una innovación social. 
4.2 ASUNTOS RELEVANTES PARA LAS MUJERES Y APORTES DE LA RED EN EL DESARROLLO SOSTENIBLE

Figura 4. Relación entre los asuntos relevantes para las mujeres y la contribución percibida que la red genera en cada tema relevante

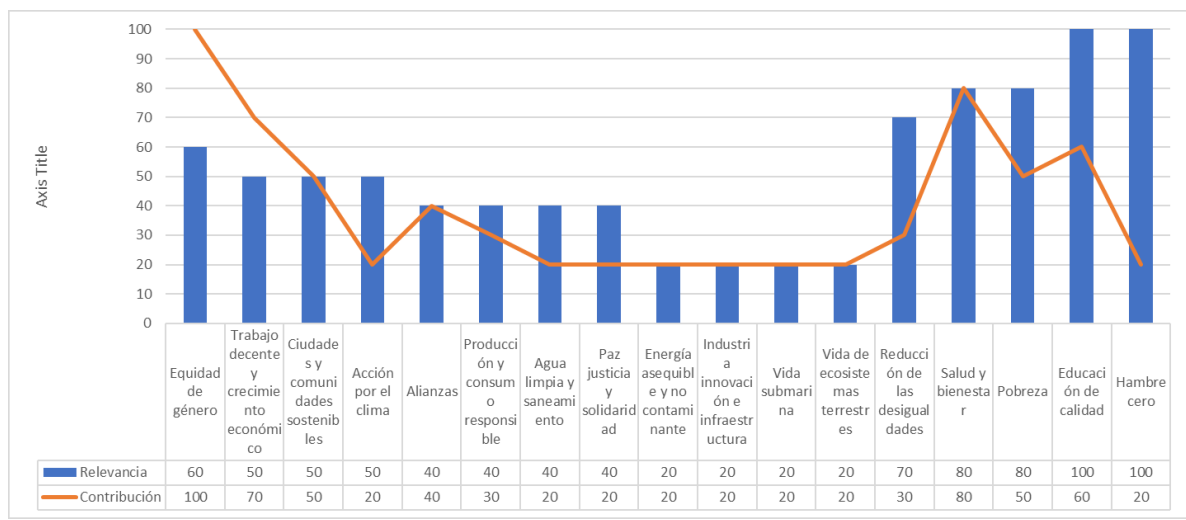

Fuente: Elaboración propia.

Se clasificaron los asuntos relevantes en los 17 objetivos de desarrollo sostenible para tener una referencia y marco de comparación.

En los resultados vemos que los principales aportes percibidos de las redes en materia de desarrollo sostenible se encuentran en equidad de género y la generación de trabajo decente y crecimiento económico. Cabe destacar que, para estos puntos en particular, el beneficio generado por la red es mayor al esperado por las mujeres que las conforman.

Se observa que los temas de mayor relevancia para las mujeres son aquellos asociados con la dimensión personal de los ODS: Pobreza, hambre, educación, salud. Sin embargo, únicamente en salud y bienestar la expectativa de contribución alcanza el nivel de importancia que tiene el tema. En los demás de esta categoría la contribución de las redes está por debajo de lo esperado según la relevancia que tienen los temas para las mujeres.

El grupo de asuntos relevantes de menor relevancia para las mujeres son los asociados a la categoría planeta. Donde solo acción por el clima alcanza el $50 \%$ de relevancia. Este es un punto alarmante, ya que, en un estudio de abril 2020, se demostró que el cambio climático y los fenómenos naturales exacerban la desigualdad entre hombres y mujeres. (Naciones Unidas, 2020) 
4.3 APORTES INDIVIDUALES Y LIMITACIONES DE LA RED PARA EL INDIVIDUO

Figura 5. Frecuencia de beneficios mencionadas por las entrevistadas

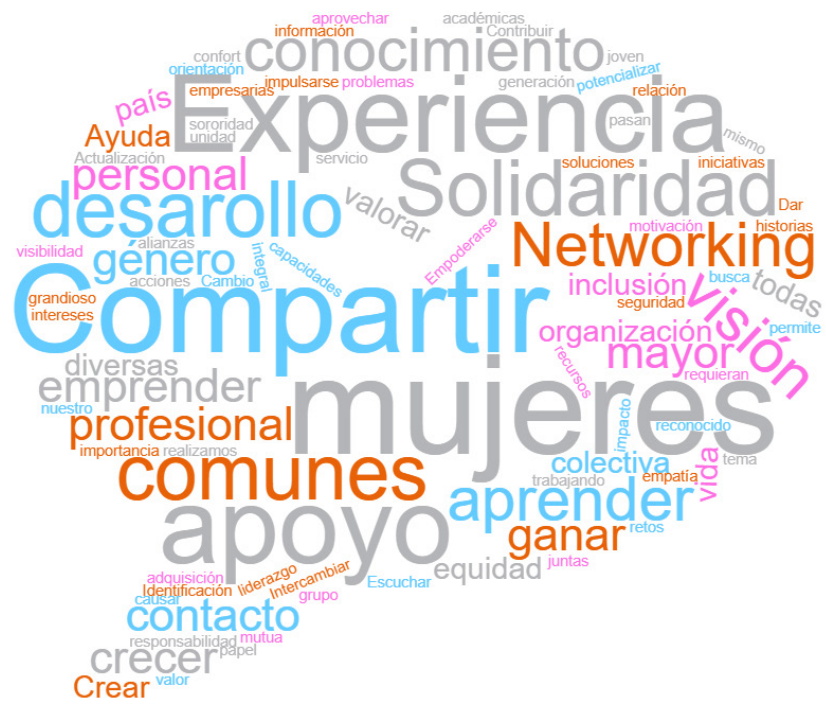

Fuente: Elaboración propia, 2020

Al transcribir las respuestas brindadas por las mujeres con relación a los beneficios de las redes de colaboración mujeres, compartir, experiencias, solidaridad y apoyo son las palabras con mayor frecuencia. Si se categorizan los beneficios mencionados, se identifican 3 beneficios principales:

- Adquirir nuevas experiencias/habilidades: El 53\% afirma que participar en redes de colaboración de muejeres le permite adquirir habilidades y experiencias que contribuyen a su bienestar personal y un $50 \%$ considera que las habilidades y experiencias adquiridas le ayudan también en el trabajo.

- Contar con el acompañamiento para la resolución de problemas: Un $56.8 \%$ de las mujeres afirman que se sienten más seguras en la toma de decisiones al estar acompañadas y contar con la visión de otros que afrontan las mismas situaciones. El mismo porcentaje de mujeres, considera que su participar en redes de colaboración de muejeres tiene un beneficio para el desarrollo del país. 
- Capital Social: El 51\% de las mujeres hizo referencia a cómo participar en red promueve la toma de decisiones colaborativa y por tanto el consenso colectivo de los temas que aquejan a la sociedad.

- La única limitante mencionada por las participantes es el tiempo y horarios, asunto este que es clave en la equidad de género.

- Sin embargo, se logró identificar que no hay claridad a lo largo de las redes del concepto de equidad de género y que no todas las participantes tienen claridad del alcance de su rol en la red.

\subsection{OTROS HALLAZGOS}

- $96 \%$ de las participantes perciben una fuerte solidaridad en su red de colaboración. El 4\% restante menciona que la solidaridad se construye en el camino.

- Solo un $2 \%$ de las mujeres consideraron luego de explicarles que es la innovación social, que ésta no está asociada a las redes de mujeres. Las demás consideran que su red trabaja para encontrar soluciones novedosas a problemas latentes y relevantes para ellas y que estas soluciones se cocrean de manera democrática y participativa.

\section{CONSIDERACIONES FINALES}

Con este artículo, la autora intuye el aporte que las redes de colaboración de mujeres en Honduras tienen en el desarrollo sostenible y en la búsqueda de soluciones innovadoras a problemas sociales, mediante la generación de capital social y la solidaridad. Se pretende brinde una primera mirada a las redes de colaboración social en Honduras, misma que se puede complementar con trabajos de investigación posteriores sobre cada tipo de red de colaboración de mujeres y particularmente se recomienda profundizar la oportunidad existente en las redes de colaboración para estudiantes.

\section{BIBLIOGRAFÍA}

Bertoni, R., Castelnovo, C., y Cuello, A. (2011).¿Qué es el desarrollo? ¿Cómo se produce? ¿Qué se puede hacer para promoverlo? Construcción y análisis de problemas del desarrollo. Montevideo: Udelar.

Cardesa-Salzmann, A., y Pigrau, A. (2017). La agenda 2030 y los onjetivos para el desarrollo sostenible. Una mirada crítica sobre su aportación a la gobernanza global en térmicos de justicia distributiva y sostenibilidad ambiental. Revista Española de Derecho Internacional, 279-285.

CEPAL. (2014). Caminos hacia la equidad de género en América Latina y el Caríbe. 9a Conferencia Regional de la Mujer En America Latina y el Caribe. CEPAL. 
Chaker, A. (2018). Sin igualdad de género no se alcanzarán los objetivos de desarrollo sostenible de la agenda 2030. Comercio Global, Camara de comercio e industrias de TEgucigalpa, 34-37.

Christensen, C. M., Braumann, H., Ruggles, R., y Sadtler, T. M. (2006). Disruptive Innovation for Social Change. Harvard Business Review, 1.

COHEP. (2019). Principales Indicadores Socio Laborales 2019. Obtenido de Consejo Hondureño de la Empresa Privada: http://cohep.com/?smd_ process_download=1ydownload_id $=8445$

Easterly, W. (2015). El problema de los objetivos de desarrollo sostenible. Current History, 322-324.

Foro económico Mundial. (2016). Foro económico Mundial. Davos: Foro económico Mundial.

Foro Económico Mundial (2020). Global Gender Gap 2019. Obtenido de Foro Económico Mundial: http://www3.weforum.org/docs/WEF_ GGGR_2020.pdf

Herrero de Egaña, B. (2019). Social Innovation, Social Entrepreneurship and Gender: Women's Approach to Facing Relevant Social Challenges. 2nd International Conference on (págs. 327-335). Roma: Academic Conferences and Publishing International Limited.

Herrero de Egaña. (2018). La Innovación Social en España: Ejes Vertebradores desde la Teoría Fundamentada. Universidad Pontíficia de Comillas, 268.

Herrero de Egaña, B. (2018). La innovación social en España: ejes vertebradores desde la Teoría Fundamentada. Tesis Doctoral, en https:// www.researchgate.net/publication/339799564_La_Innovacion_Social_ en_Espana_Ejes_vertebradores_desde_la_Teoria_Fundamentada.

INE. (2020). Población en Honduras. Tegucigalpa: INE.

Instituto Nacional de Estadísticas. (5 de noviembre de 2020). Población en Honduras. Obtenido de Instituto Nacional de Estadísticas: https://www. ine.gob.hn/V3/

Lampert, M. (2014). BCN Minuta: Concepto equidad de género. Santiago: Biblioteca del Congreso Nacional de Chile.

Lean in circle. (2020). About Lean in circle. Obtenido de Lean in circle: https://leanin.org/about

Lifeder. (2020). Obtenido de https://www.lifeder.com/sensibilidad-social/ Muñoz, H., y Sánchez, J. (2018). Responsabilidad e innovación social. En H. Muñoz, J. Sánchez, Responsabilidad Social: Lecturas y debates (págs. 1329). Antioquia: Fondo Editorial Universidad Católica de Oriente.

Naciones Unidas. (25 de Septiembre de 2015). Objetivos de Desarrollo Sostenible. Obtenido de Naciones Unidas: https://www.un.org/ sustainabledevelopment/es/ 
Naciones Unidas. (2016). Conferencia Mundial por el Clima. Conferencia Mundial por el Clima. Bonn: Naciones Unidas.

Naciones Unidas. (2020). Agenda 2020: Igualdad de género para no dejar a nadie atrás. Obtenido de Programa de Naciones Unidas para el Desarrollo: http://americalatinagenera.org/newsite/index.php/es/ igualdad-de-genero-y-agenda-2030

Naciones Unidas. (29 de abril de 2020). Portal de noticias Naciones Unidas. Obtenido de Naciones Unidas: https://news.un.org/es/ story/2020/04/1473642

OIT. (enero de 2020). Panorama Laboral 2019: América Latina y el Caribe. Obtenido de OIT: https://www.ilo.org/wcmsp5/groups/public/--americas/---ro-lima/documents/publication/wcms_732198.pdf

OMS. (2002). Política de la OMS en materia de género: Integraciónd e las perspectivas de género en la labor de la OMS. Obtenido de OMS: https://www.who.int/teams/gender-equity-and-human-rights

ONU. (1987). Reporte de La comisión Mundial de Ambiente y Desarrollo: Nuestro Futuro Común. Oslo: ONU. Obtenido de https:// sustainabledevelopment.un.org/content/documents/5987ourcommon-future.pdf

ONU. (1992). Cumbre de la Tierra. Río de Janeiro: ONU.

OWSD. (14 de noviembre de 2020). OWSD. Obtenido de OWSD: https:// owsd.net/about-owsd/what-owsd

Pérez, J., Merino, M. (2009). Definiciones . Obtenido de Definiciones : https://definicion.de/equidad-de-genero/

PNUD. (2019). Informe sobre Desarrollo Humano 2019: Desigualdad del desarrollo humano en el siglo XXI. Tegucigalpa: Naciones Unidas.

PNUD. (2019). Participación de la mujer para el desarrollo de Honduras. Tegucigalpa: PNUD.

Portela, M., Neira, I. (2003). Capital social: las relaciones sociales afectan el desarrollo. Chorlavi.

RAE. (2020). Diccionario de la lengua española. RAE.

Rodríguez, I., Govea, H. (2006). El discurso del desarrollo sustentable en America Latina . Revista Venezolana de Economía y Ciencias Sociales, 37-73.

Tribunal Supremo Electoral. (2017). Tribunal Supremo Electoral. Obtenido de Estadísticas Elecciones Generales 2017: https:// eleccionesgenerales2017.tse.hn/

UNESCO. (2014). Plan de acción prioritario sobre igualdad de género de la UNESCO: 2014-2021. Obtenido de UNESCO: https://unesdoc.unesco. org/ark:/48223/pf0000227222

Voces Vitales. (14 de noviembre de 2020). Voces Vitales. Obtenido de https://vocesvitaleshonduras.org/nosotros/primer-paso-para-crecer/ 